\title{
Ultrasensitive FRET Based DNA Sensor Using PNA/DNA Hybridization
}

\author{
Eunhae Koo, Lan-Hee Yang \\ Korea Institute of Ceramic Engineering and Technology \\ 101, Soho-ro, Jinju-City, Republic of Korea \\ ehkoo@kicet.re.kr; yangd5d5@korea.ac.kr
}

\section{Extended Abstract}

In the diagnosis of genetic diseases, rapid and highly sensitive DNA detection is crucial. Therefore, many strategies for detecting target DNA have been developed, including electrical, optical, and mechanical methods [1][2][3]. We developed a DNA sensor with femtomolar sensitivity based on Förster resonance energy transfer (FRET) [4][5]. We use a peptide nuclear acid (PNA) probe, which is an artificially created nucleic acid that exhibits greater sensitivity and stability than DNA. Moreover, we used quantum dots (QDs) as donors in the FRET system because QDs exhibit high quantum yields [6][7].

In this study, we developed a DNA nanosensor with picomolar sensitivity based on Förster resonance energy transfer (FRET) using quantum dots (QDs) as donors. 620nm QDs are linked with hybridized PNA/DNA of capture probe, reporter probe and target probe. The hybridized probe with wild type's target DNA has Cy5 fluorescence signal due to FRET from QD having high quantum yield about $70 \%$. $\mathrm{COOH}-\mathrm{NH}_{2}$ linking system turns out to be high FRET efficient than avidin-biotin linking system in the hybridized PNA/DNA systems. Based on the FRET signal in low molar ratio of acceptor to donor fluorophore $(\mathrm{R}=\mathrm{Cy} 5 / \mathrm{QD})$, the results indicate that PNA based probe is much more sensitive than DNA based probe.

Keywords: Förster resonance energy transfer (FRET), QD, DNA sensor, microcapillary, peptide nuclear acid (PNA)

\section{References}

[1] C.-Y. Zhang, H.-C. Yeh, M. T. Kuroki, and T.-H. Wang, "Single-quantum-dot-based DNA nanosensor," nature materials, vol. 4, pp. 826-831, 2005.

[2] H. Kuhn, "Hybridization of DNA and PNA Molecular Beacons to Single-Stranded and Double-Stranded DNA Targets," J. AM. CHEM. SOC., vol. 124, no. 6, pp. 1097-1103, 2002.

[3] A. P. Alivisatos, "Semiconductor Clusters, Nanocrystals, and Quantum Dots," Science, vol. 271, pp. 933-937, 1996.

[4] G. Jiang, A. S. Susha, A. A. Lutich, F. D. Stefani, J. Feldmann, and A. L. Rogach, "Cascaded FRET in conjugated polymer/quantum dot/dye-labeled DNA complexes for DNA hybridization detection," ACSnano, vol. 3, no. 12, pp. 4127-4131, 2009.

[5] C.-Y. Zhang and L. W. Johnson, "Microfluidic control of fluorescence resonance energy transfer: Breaking the FRET limit," Angew. Chem. Int. Ed., vol. 46, pp. 3482-3485, 2007.

[6] O. Brandt, J. D. Hoheisel, "Peptide nucleic acids on microarrays and other biosensors," Trends in Biotechnology, vol. 22, pp. 617-22, 2004.

[7] H. Kuhn, "Hybridization of DNA and PNA Molecular Beacons to Single-Stranded and Double-Stranded DNA Targets," J. AM. CHEM. SOC., vol. 124, no. 6, pp. 1097-1103, 2012. 